$P \& A$ Año 3, N. ${ }^{\circ}$

enero-junio 2018

pp. [55]-65

\title{
Resumen
}

La calidad de educación, como base de desarrollo de una sociedad progresivamente introducida en un contexto globalizado, exige a reflexionar sobre la manera en la cual se forma a los futuros profesionales

Con el objetivo de mejorar la calidad del servicio educativo, el Estado peruano, comienza a normar la creación de universidades, licenciamiento para su funcionamiento, supervisión a partir de la evaluación, acreditación y certificación, medida que en caso desaprobatorio puede llegar incluso al cierre de las universidades con la Ley 30220.

El caso que se presenta corresponde a la asignatura de Urbanismo I, del área académica de urbanismo de la carrera de Arquitectura, la cual toma los factores de análisis definidos por la Acreditadora internacional CINDA-IAC en su modelo de autoevaluación, pero referidas al Plan de Estudios y que en esta oportunidad son aplicados en el desarrollo de los trabajos prácticos de la asignatura, del cuarto ciclo de estudios.

Palabras clave: Calidad Educativa, objetivos de aprendizaje, acreditación internacional.

\section{La Calidad educativa y los objetivos de aprendizaje Caso: Asignatura de Urbanismo I}

\author{
Arq. Soledad Guadalupe Herrera García*
}

Recibido: 18 de julio de 2017

Aceptado: 21 de enero de 2018

\begin{abstract}
Quality of education, as the basis for the development of a society progressively introduced in a globalized context, requires reflection on the way future professionals are being trained.

In order to improve the quality of the educational service, Peruvian state has begun to regulate the creation of universities, license for its operation, supervision based on evaluation, accreditation and certification. In case of disapproving, this measure can even close the universities according to Act 30220.

The case developed corresponds to Urbanism I, a subject of the urbanism area in the architecture career, which assumes the analysis factors defined by the CINDA-IAC, International Accreditation Center, in its self-assessment model but referred to the Study Plan that in this opportunity is applied to the development of the practical works of the subject belonging to the fourth cycle of studies
\end{abstract}

Keywords: : Educational Quality, learning objectives, international accreditation.

\footnotetext{
* Arquitecta por la Universidad Ricardo Palma, con estudios de maestría de Transporte Urbano y Docencia Superior, ambas en la Universidad Ricardo Palma; con experiencia en habilitaciones urbanas, construcción de edificaciones residenciales, consultoría en el área de planificación; docente del área de urbanismo de la Universidad Ricardo Palma.
} 


\section{La Globalización en la educación y los agentes de cambio}

La Educación a nivel mundial enfrenta nuevos retos para satisfacer las diferentes demandas que la sociedad solicita del quehacer educativo, y es en este afán que se desarrollan continuamente nuevos planteamientos para alcanzar una educación de calidad.

En los sistemas educativos de la mayoría de sociedades, se entiende, que el desarrollo cognitivo de valores, actitudes y destrezas son objetivos importantes; donde los contenidos pueden diferir, pero la estructura es, a grandes rasgos, similar en todo el mundo; lo que sugiere que, en cierto sentido, la clave para mejorar la calidad educativa -ayudar a los sistemas educativos a alcanzar de manera óptima estos objetivos- podría ser también mundial.

Es por este motivo que desde décadas atrás se reúnen las organizaciones internacionales para plantear nuevos enfoques educativos, muy necesarios ante los cambios que se presentan las sociedades con exigencias de calidad para los profesionales.

En 1972 la Comisión internacional sobre el Desarrollo de la Educación de UNESCO, presidido por el exministro francés Edgar Faure, manifestó que: "la meta y el contenido de la educación deben ser recreados para permitir nuevas características en la sociedad y nuevas características en la democracia”.

Luego, en 1996, la Comisión internacional sobre Educación para el siglo XXI, presidido por el estadista francés Jacques Delors, resume muy apropiadamente que la educación a lo largo de la vida se basa en cuatro pilares: Aprender a conocer, Aprender a hacer, Aprender a vivir juntos, Aprender a ser.

Configurándose, así, una mirada integradora y comprensiva del aprendizaje, y por lo tanto de aquello que constituye una educación de calidad

Se continúan los esfuerzos para avanzar, y en 2003, la UNESCO en la mesa redonda de Ministros sobre la educación de buena calidad que se sostuvo en Paris, refuerza la importancia de la buena educación en el que promueve un acceso a la educación como derecho humano y sustenta un enfoque basado en esto, y en todas las actividades educativas.

Este último, enfoque según Pigozzi (2004), comprende un aprendizaje en dos niveles, el primero a nivel de los estudiantes y el segundo a nivel del sistema de aprendizaje.

En este segundo nivel, es en el cual se plantean los alcances que permiten evaluar la consistencia interna del proceso de enseñanza aprendizaje respecto del Plan de Estudios. La orientación de este proceso, lo determina la institución educativa en su modelo pedagógico y se manifiesta en los sílabos de las asignaturas a través de las sumillas que sintetizan la esencia misma de la asignatura y sus particularidades. Es necesario identificar las competencias genéricas que se desprenden del modelo pedagógico de la institución, así como las competencias específicas que se derivan de las áreas temáticas y están definidas previamente en el Perfil de Egreso de cada Plan de Estudios que se actualiza periódicamente cumpliéndose, así, con los cambios del tipo de profesional que la sociedad requiere.

En el proceso de enseñanza aprendizaje interactúan los actores docentes y estudiantes, dentro de diferentes estrategias didácticas para alcanzar las competencias requeridas entendidas como objetivos a lograr en el proceso de transformación del estudiante para el logro del Perfil de Egreso.

\section{De la ley universitaria 30220}

La preocupación del Estado peruano para la mejora de la calidad educativa tiene larga data, pero un hito importante es cuando el Tribunal Constitucional del Perú, emite la sentencia STC 00017-2008-PI/TC emitida en el 2010 que dejó establecido en el punto resolutivo 4 la existencia de un estado de caos inconstitucional de carácter estructural, en el sistema educativo universitario y estableció como una obligación, por parte del Estado, adoptar las medidas institucionales necesarias para reformar el sistema de educación universitaria en el país, de forma tal que quede garantizado el derecho fundamental de acceso a una educación 
universitaria de calidad, derecho reconocido por la Constitución Política del Perú.

En el numeral 129 de la sentencia, citada anteriormente, se señala la necesidad de crear una Superintendencia Nacional de Educación Superior altamente especializada, objetivamente imparcial y supervisada eficientemente por el Estado, y que tiene como competencias: evaluar a todas las universidades del país existentes y sus respectivas filiales adoptando las medidas necesarias para cuando sea necesario elevar su nivel de calidad educativa, y evaluar a todas las universidades y filiales ratificadas o autorizadas por el CONAFU. Es en cumplimiento de esta sentencia que se promulga la nueva ley universitaria 30220 el 3 de julio de 2014 y con esta promulgación se crea la Superintendencia Nacional de Educación Superior Universitaria (SUNEDU) y se establece el licenciamiento obligatorio y renovable de las universidades; dejando sin efecto las autorizaciones de funcionamiento del anterior marco legal.

Posteriormente, el Decreto Supremo N. 16-2015-MINEDU aprobó la Política de Aseguramiento de la calidad de la Educación Superior Universitaria, la misma que establece el licenciamiento obligatorio y se constituye en un mecanismo de protección del bienestar individual y social de los ciudadanos que buscan acceder al Sistema Universitario.

En el contenido de la ley universitaria, se resalta

Capítulo III: Creación y licenciamiento de universidades. Artículo 28: Licenciamiento de universidades. Las condiciones básicas que establezca la SUNEDU para el licenciamiento, están referidas como mínimo a los siguientes aspectos: $28.1 \mathrm{La}$ existencia de objetivos académicos, grados y títulos a otorgar, así como los planes de estudio correspondientes. 28.2 Previsión económica y financiera de la universidad a crearse compatible con los fines propuestos en sus instrumentos de planeamiento. 28.3 Infraestructura y equipamiento adecuados al cumplimiento de sus funciones (bibliotecas, laboratorios, entre otros). 28.4 Líneas de investigación a ser desarrolladas. 28.5
Verificación del personal docente calificado con no menos del $25 \%$ de docentes a tiempo completo. 28.6 Verificación de los servicios educacionales complementarios básicos (servicio médico, social, psicopedagógico, deportivo, entre otros). 28.7 Existencia de mecanismos de mediación e inserción laboral (bolsa de trabajo u otros).

Es necesario el cumplimiento de las normas vinculadas a la mejora de la calidad en el servicio educativo y en este sentido la ley universitaria considera:

Capítulo IV: Evaluación, Acreditación y Certificación. Artículo 30: Evaluación e incentivo a la calidad educativa El proceso de acreditación de calidad educativa en el ámbito universitario, es voluntario, se establece en la ley respectiva y se desarrolla a través de normas y procedimientos estructurados funcionalmente. Los criterios y estándares que se determinen para su cumplimiento, tienen como objetivo mejorar la calidad en el servicio educativo. Excepcionalmente, la acreditación de la calidad de algunas carreras será obligatoria por disposición legal expresa.

\section{Proceso de acreditación internacional y nacional}

Los procesos de acreditación internacional orientan sus modelos siguiendo pautas de la UNESCO y para la acreditación nacional se rigen por lo dispuesto por la Superintendencia Nacional de Educación Superior Universitaria (SUNEDU) que le confiere la ley universitaria 30220.

En estos procesos, lo vital es constatar el servicio educativo de calidad que ofrece la institución de educación superior universitaria y para ello se valen de dimensiones, variables, factores, estándares, etc. Según sea la estructura del modelo que emplee la agencia acreditadora internacional, y en el caso de la acreditación nacional se trabaja con el nuevo modelo de SUNEDU que comprende la nueva matriz de evaluación que está organizada en 4 dimensiones, 12 factores y 34 estándares. 
Tanto en la acreditación nacional como en la internacional, la institución educativa encarga a cada Carrera la realización de la autoevaluación con la participación, involucramiento y compromiso de sus estamentos, declarando un diagnóstico de situación que se constata con una visita de evaluación de Pares académicos, que informan de la verificación de lo actuado para la determinación del dictamen de acreditación cuando corresponda. Entendiéndose que esta, será continuamente verificada por la institución o agencia acreditadora.

\section{Estudio de Caso en la asignatura de Urbanismo I del cuarto semestre de la Carrera de Arquitectura de la Facultad de Arquitectura y Urbanismo-URP}

El modelo de CINDA-IAC, agencia que acredita internacionalmente las carreras universitarias, ha organizado una estructura de modelo que lo conforman: Dimensiones, factores y criterios. Modelo de evaluación validado por expertos.

Para este caso se trabaja la Dimensión I: Perfil de Egreso y resultados, la carrera tiene un perfil de egreso claramente formulado, actualizado en función de los elementos disciplinarios, profesionales y laborales correspondientes, y consistente con las orientaciones de la misión institucional. Dicho perfil contempla explícitamente las competencias asociadas al título y/o grado académico otorgado.

El Factor: 1.2 Estructura curricular, donde se establece que el currículo de la carrera está estructurado en función del perfil de egreso previamente definido, considerando tanto las competencias directamente vinculadas al desempeño profesional como las de carácter general y complementario.

Es en el Criterio 8, que se caracteriza por la esencia del caso a tratar, y donde establece claramente que: el Plan de estudios contempla explícitamente objetivos del aprendizaje de carácter general, tales como:

Interacción social: Capacidad para formar parte de equipos de trabajo y participar en proyectos grupales
Pensamiento globalizado: Capacidad para comprender los aspectos interdependientes del mundo globalizado.

Pensamiento crítico: Capacidad para utilizar el conocimiento, la experiencia y el razonamiento para emitir juicios fundados.

Solución de problemas: Capacidad para identificar problemas, planificar estrategias $y$ enfrentarlos.

Autoaprendizaje e iniciativa personal: Inquietud y búsqueda permanente de nuevos conocimientos y capacidad de aplicarlos y perfeccionar sus conocimientos anteriores.

Formación y consistencia ética: Capacidad para asumir principios éticos y respetar los principios del otro, como norma de convivencia social.

Sensibilidad estética: Capacidad de apreciar y valorar diversas formas artísticas y los contextos de donde provienen.

Formación ciudadana: Capacidad para integrarse a la comunidad y participar responsablemente en la vida ciudadana.

Comunicación: Capacidad para comunicarse de manera efectiva a través del lenguaje oral y escrito, y del lenguaje técnico y computacional necesario para el ejercicio de la profesión.

\section{Estudio de Caso: Asignatura Urbanismo I, cuarto semestre de la Carrera de Arquitectura.}

Primero, es conveniente explicar que el proyecto pedagógico de la carrera, el enfoque de Competencias, la estructura del Plan de Estudios 2015, la secuencia de asignaturas y los sílabos. Este conjunto interrelacionado que orienta las estrategias de aprendizaje y los instrumentos de evaluación elaborados para cada ejercicio que se desarrolla se constituyen en el binomio competencia-evaluación continua, que sintetiza la obtención de las piezas clave del perfil de Egreso.

Los objetivos de carácter global del modelo CINDA-IAC son aplicados en este caso a una asignatura y comprenden: 

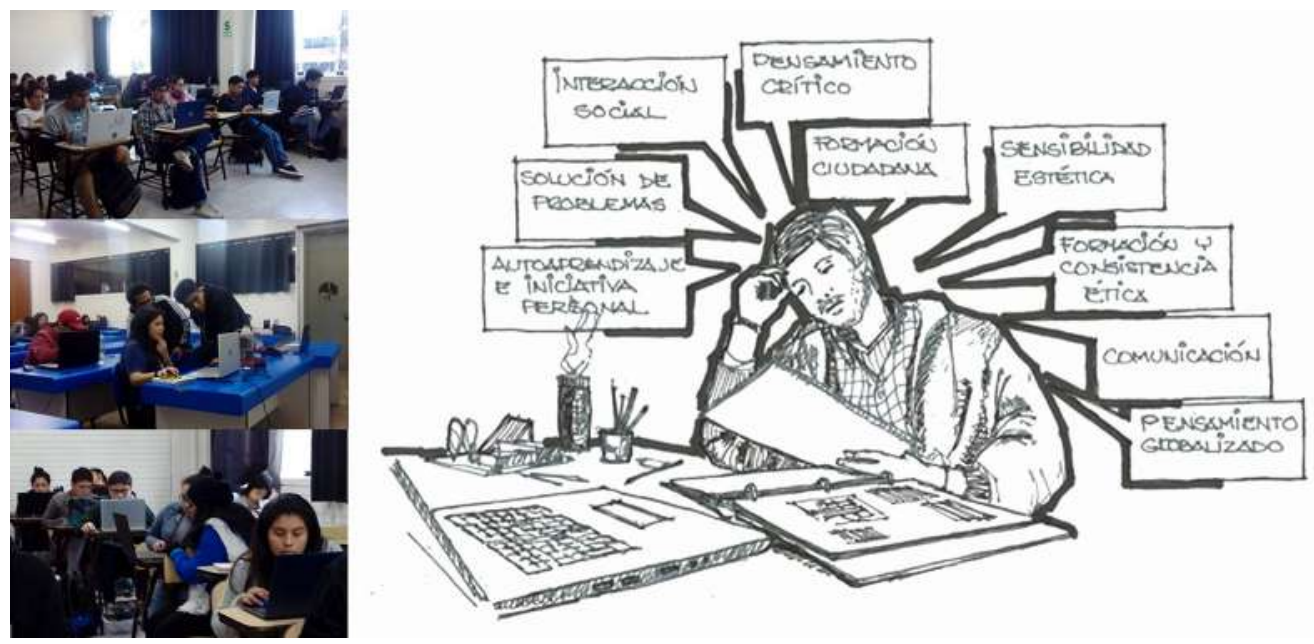

Figura 1. Los objetivos de aprendizaje de caracter general y sus aplicación en la asignatura Urbanismo I de la Facultad de Arquitectura de la Universidad Ricardo Palma (2018).

\section{Interacción social}

Entendida como una característica fundamental en la vida, es decir, todos los individuos obligatoriamente interactúan con otros diariamente, virtual o físicamente, excepto aquellos que decidieron ser monjes o vivir verdaderamente como ermitaños; es el proceso por el cual uno actúa y reacciona a quienes lo rodean e incluye los actos que las personas realizan entre sí, y las respuestas que dan a cambio. La microsociología, creada por Erving Goffman, investiga, analiza e intenta comprender la vida social a través de las interacciones de las personas y la forma en que lo hacen.

De acuerdo con el orden social, una regla efectiva para hacer que esta sociedad funcione bien es la interacción social efectiva y Goffman Erving (1993) distingue dos tipos principales de interacción:

a. Interacción enfocada, que indica interacción entre un grupo de personas que tienen un objetivo común, estas pueden haber estado familiarizadas entre sí en el pasado o pueden haberse familiarizado en el primer momento de su interacción enfocada. Un ejemplo de esto es un grupo de jóvenes que estudian juntos para un examen final, un equipo de fútbol o los asistentes a un concierto. b. Interacción no enfocada, no incluye ningún objetivo común o familiaridad, incluso durante el proceso de interacción. De hecho, las personas que interactúan pueden no ser conscientes de su interacción, como por ejemplo la interacción entre los peatones que evitan las colisiones desastrosas que siguen las señales y regulaciones de tráfico.

Aplicando al caso, la interacción enfocada entre los estudiantes de la asignatura para lograr un objetivo común; donde la libertad de conformar grupos de trabajo, de cuatro integrantes y de seleccionar su área de estudio, implica ponerse de acuerdo para elegir.

La atención y concentración de lo que se va a trabajar implica interacción constante entre los integrantes, así como alcanzar la comprensión de la secuencia de las actividades a cumplir y los requisitos explicados durante la sesión.

Por parte del facilitador; este debe configurar un listado de temas de acuerdo a la cantidad de grupos que conforman los estudiantes de la asignatura. Los avances logrados en el desarrollo del trabajo son compartidos en cuanto a las referencias electrónicas, los aciertos y desaciertos producidos durante el proceso son presentados por el facilitador a todo el grupo para fortalecerlo. Los logros obtenidos por al- 
gún equipo de trabajo y exponer a todos, los desaciertos que impiden el avance o trabajo mal encaminado.

La interacción social entre los estudiantes se propicia constantemente tanto a nivel de integrante de equipo o como acompañamiento del facilitador, cuando trabaja conjuntamente en la pizarra o en su computadora realizando esquemas, gráficos, tablas, etc. Actividades que le permiten explicar a sus compañeros de asignatura respecto de lo trabajado en conjunto con una actitud de seguridad y confianza que influye en el mejoramiento de su autoestima.

La interacción social es indispensable para los estudiantes de la carrera de Arquitectura, ya que una de las características de la profesión es la continua interacción con los diferentes usuarios que requieren sus servicios profesionales, y cada uno de ellos con diferentes visiones del mundo y personalidad, que debe entender el profesional de la carrera para interactuar y comprender los requerimientos de la obra solicitada.

\section{Pensamiento globalizado}

La actitud del ser humano que se basa en la percepción del mundo real en término de totalidades para su análisis, comprensión y accionar. Este pensamiento es integrador tanto en el análisis como en las conclusiones que nacen a partir de allí, proponiendo soluciones considerando elementos diversos y relaciones que conforman la estructura de lo que se define como sistema.

Este pensamiento tiene cualidades únicas que lo hace una herramienta invaluable que enfatiza la observación del todo y no de sus partes. Es un lenguaje circular que enfatiza en la retroalimentación en lugar de las causas y efectos lineales, convierte las percepciones individuales en imágenes explícitas que dan sentido a los puntos de vista de cada persona involucrada.

La capacidad para comprender la interdependencia que existe entre los países del mundo. Tal es el caso de los aportes de otros países en cuanto a la búsqueda de métodos, innovaciones tecnológicas, enfoques, etc.
Hoy en el campo educativo se emplean herramientas pedagógicas, como los casos y los simuladores, los ejecutivos, por ejemplo, prefieren programas que les brindan conocimientos más prácticos y les permitan potenciar sus habilidades blandas como la capacidad de liderazgo, el trabajo en equipo y la buena comunicación porque es lo que el mercado globalizado exige.

En el aula, el facilitador emplea un método de trabajo que proviene de una organización supranacional, incorporando así al estudiante en una interacción con métodos provenientes de otras realidades y entender la visión de expertos internacionales para analizar el territorio y sus complejidades.

En este método denominado por PNUD "Sistema territorial" que comprende cuatro subsistemas: subsistema biofísico, sociocultural, económico institucional y construido, donde las particularidades de cada uno de ellos se trabajan siguiendo las pautas seleccionadas para este nivel de estudios (cuarto semestre).

\section{Pensamiento crítico}

Robert Ennis (1985). Este autor lo concibe como el pensamiento racional y reflexivo interesado en decidir qué hacer o creer. Es decir, por un lado, constituye un proceso cognitivo complejo de pensamiento que reconoce el predominio de la razón sobre las otras dimensiones del pensamiento. Su finalidad es reconocer aquello que es justo y aquello que es verdadero, es decir, el pensamiento de un ser humano racional. El desarrollo del pensamiento crítico se facilita con el aprendizaje basado en problemas, que en el mismo proceso de enseñanza-aprendizaje no lo incorpora como algo adicional, sino que es parte del mismo proceso de interacción para aprender. Ese aprendizaje busca que el alumno comprenda y profundice adecuadamente en la respuesta a los problemas que se usan abordando aspectos de orden filosófico, sociológico, psicológico, histórico, práctico, etc. Todo lo anterior con un enfoque integral donde la estructura y el proceso de solución al problema están siempre abiertos, lo cual motiva a un aprendizaje consciente y al trabajo de grupo sistemático en una experiencia colaborativa de aprendizaje. 
Este trabajo colaborativo se aplica en el desarrollo del trabajo, al usar el conocimiento previo y fuentes de información que han sido trabajadas por instituciones del Estado peruano, tales como IGN, INEI, SERNANP, entre otras y lograr que el estudiante construya una data en Excel respecto del tema abordado donde se describe, luego se explica y, finalmente, se obtienen conclusiones de la tabla elaborada. (Data del área de estudio)

Otro aspecto que se desarrolla, es de la lectura gráfica que se obtiene con la utilización de colores, texturas, símbolos estandarizados y símbolos creados ad hoc con intención de obtener legibilidad gráfica; se resaltan valoraciones cualitativas o cuantitativas, que permiten emitir juicios fundados sobre lo trabajado, desarrollo que debe ser incrementado poco a poco en los trabajos de la asignatura.

\section{Solución de Problemas Método}

Tiene sus primeras aplicaciones y desarrollo en la escuela de medicina en la Universidad de Case Western Reserve en los Estados Unidos y en la Universidad de McMaster en Canadá en la década de los 60. Desde esta fecha su uso se ha extendido a diversas universidades. Esta se sustenta en diferentes corrientes teóricas sobre el aprendizaje humano, tiene particular presencia la teoría constructivista, de acuerdo con esta postura en el ABP se siguen tres principios básicos: - El entendimiento con respecto a una situación de la realidad que surge de las interacciones con el medio ambiente.

- El conflicto cognitivo al enfrentar cada nueva situación estimula el aprendizaje.

- El conocimiento se desarrolla mediante el reconocimiento y aceptación de los procesos sociales y de la evaluación de las diferentes interpretaciones individuales del mismo fenómeno.

En las horas de práctica de asignatura se presenta la oportunidad para identificar problemas la que se manifiesta muy bien en los trabajos en clase, usando el método FODA aplicado al análisis territorial que permite presentar un diagnóstico de situación en términos de fortalezas y debilidades; desarrollando así sus capacidades de análisis, síntesis e investigación; además los estudiantes agrupados desarrollan habilidades para trabajar en grupo, emiten juicios de valor; desarrollan sus habilidades comunicativas para avanzar hacia la prognosis y continuar hacia la formulación de planes. Asumen con responsabilidad su propio aprendizaje y dejan que el tutor les oriente en la adquisición de conocimiento.

El facilitador incluye, dentro de la estrategia de aprendizaje, el "corta y pega" que los estudiantes practican erróneamente y que se convierte en la herramienta de la fase final del trabajo práctico. Con este método se busca en el portal de proyectos de inversión del gobierno, una matriz de impacto ambiental y la localización espacial del proyecto; la matriz es utilizada parcialmente incorporando un método de valoración de impactos ambientales muy simple proporcionado por el facilitador, iniciando con actitud positiva esta estrategia de aprendizaje viable y aplicable para lograr los objetivos de aprendizaje.

\section{Autoaprendizaje e iniciativa personal}

Es reconocida por una competencia básica de las básicas.

A finales de los noventa del siglo XX, la Organización para la Cooperación y el Desarrollo Económico (OCDE) realizó un estudio en doce países de su entorno para determinar cuáles serían realmente las competencias clave necesarias para una vida plena y para el buen funcionamiento de la sociedad. (OCDE, 2001, pág.4)

En la publicación del Informe DeSeCo (Definición y Selección de Competencias) de 2001 se establecen tres categorías de competencias; la primera referida a usar un amplio rango de herramientas de manera interactiva en el ambiente; tanto física como en la tecnología de la información y en el uso del lenguaje; adaptarlas a sus fines y de manera interactiva.

La segunda competencia clave está referida a interactuar en grupos heterogéneos (de diversos orígenes). Y la tercera esta referida a la responsabilidad que tienen los individuos de manejar sus propias vidas, actuar de manera autónoma y situar sus vidas en un contexto social más amplio. 
Posteriormente los Consejos de Estocolmo (2001) y Barcelona (2002) establecieron los objetivos que debían cumplir los sistemas educativos y de formación europeos, y definieron un programa de trabajo, denominado "Educación y Formación 2010". Como soporte a este programa se elaboró el documento "Competencias clave para un aprendizaje a lo largo de la vida" (2004).

En este documento la Unión Europea identifica las ocho competencias clave, entre las cuales aparece el "espíritu emprendedor", que se define como "la habilidad de la persona para transformar las ideas en actos. Está relacionado con la creatividad, la innovación y la asunción de riesgos, así como con la habilidad para planificar y gestionar proyectos con el fin de alcanzar objetivos". A la vista de esta definición se entiende por qué se considera "el espíritu emprendedor" como el antecedente de la competencia para autonomía e iniciativa personal". (Gobierno Vasco, s.f., p.6)

La motivación intrínseca incrementada por la vocación a la carrera de Arquitectura impulsa al estudiante en su afán por un aprendizaje fuera del aula; es así que consulta en diferentes repositorios virtuales para obtener mayor información que la presentada en el sílabo de la asignatura.

La iniciativa personal del estudiante se propicia con las tareas opcionales que se presentan en cuatro sesiones durante el semestre, lo que permite al estudiante profundizar la temática que se desarrolló en la sesión de clase, contribuyendo así en la ampliación de sus conocimientos sobre el tema y el vocabulario correspondiente.

El facilitador percibe el interés del estudiante cuando participa en el desarrollo de la tarea opcional y se incluye en la nota de participación.

\section{Formación y consistencia ética}

Según Javier Galdona (s.f.), la formación de la estructura ética de la persona implica un proceso de construcción del cual podemos apun- tar algunas líneas de trabajo necesarias para el desarrollo del sujeto ético:

a) Aprender a clarificar lo que "cree", lo que "siente", lo que "puede". Así, mediante el desarrollo de esta capacidad en la persona, entre otras consecuencias, se evitará en gran medida: la confusión entre deber y sentimiento (con toda la carga de culpabilizaciones no adecuadas que la persona psicológicamente puede desarrollar), el voluntarismo (con su secuela de frustración) y, sobre todo, la sensación de un relativismo subjetivista que paraliza desde el punto de vista ético y que termina generando desmoralización en el sujeto. (párr. 28)

b) Aprender a no auto justificarse. El ser humano normalmente necesita buscarles una justificación plausible a sus actos, tanto ante sí mismo como ante los demás. El problema radica en la objetividad y adecuación a la realidad de esas justificaciones, es decir, en que en realidad esos actos no sean justos (adecuados a la realidad) o que esa justificación no sea plausible. (párr. 29)

Enfrentar la verdad en las diferentes circunstancias de la vida requiere coraje, cualidad que no se cultiva adecuadamente y que implica el tener un sentido de autocrítica desarrollado, así como de aceptación de sí mismo con los diferentes niveles de inconsistencia e incoherencia de los propios actos.

c) Aprender a buscar la verdad. La verdad no es autoevidente ni unívoca en la realidad histórica donde se desenvuelve el ser humano. Debe ser buscada trabajosamente, asumiendo el esfuerzo, las incertezas, las crisis personales y los momentos de claridad y obscuridad que el proceso implica. Buscar la verdad exige decisión, coraje, así como también instrumentos y habilidades que la hagan posible. Aprender a buscar la verdad supone el desarrollo de la capacidad real de diálogo, es decir, aprender a confrontar con otros las propias certezas y las propias dudas, mediante argumentaciones consistentes y con capacidad de interacción intelectiva, especialmente con aquellos que tienen perspectivas conceptuales distintas. La construcción de certezas sólo será 
abierta en la medida en que dichas certezas puedan ser confrontadas y sostenibles, a juicio del propio sujeto, ante otras posturas contradictorias con la suya. (Galdona, s.f., párr. 30)

d) Aprender a discernir entre las diferentes guías de valor en una sociedad plural. Frente a los conflictos socio-morales que la persona debe afrontar, la sociedad ofrece una variedad de guías de valor o criterios morales, cada uno de los cuales supondrá previsiblemente diferencias en el resultado final respecto de las demás. La persona necesita aprender a calibrar las diferentes propuestas éticas que recibe, a efectos de discernir cuál o cuáles de esas guías de valor son las que más condicen con sus certezas fundamentales. Esto implicará que la persona sea capaz de distinguirlas, que sea capaz de inferir los elementos antropológicos fundamentales que subyacen a cada una y, finalmente, que sea capaz de proyectar sus resultados. (Galdona, s.f., párr. 31)

En este sentido la capacidad que asumen los estudiantes para contribuir con responsabilidad en el equipo de trabajo, intercambiar lo que han elaborado a partir de fuentes de información confiable cumpliendo así con el proceso de aprendizaje y respetar la participación de cada uno de los integrantes del equipo, interactuando con respeto en esta participación conjunta. Participación de grupo, con ideas y actividades dentro de una organización determinada por ellos y dentro de un cronograma establecido.

\section{Sensibilidad estética}

Child (1965) se refiere al "grado en el cual una persona manifiesta evidencia de responder a los estímulos relevantes en alguna relación consistente y apropiada a los patrones externos". (Citado en: Manas, 1972, p. 222). Raychaudhuri Manas, desarrolla una investigación sobre el concepto definido antes por Child y considera que sensibilidad estética, medida en términos de apreciación estética, deriva en buen grado de la observación que uno tenga de las obras de arte y de la formulación que uno haga de sus propios juicios sobre ellas, de manera tal que el observador cuidadoso tiende a llegar independientemente a juicios de valor similares a aquellos a los cuales han llegado los expertos siguiendo el mismo proceso.

\section{Otros definen como:}

La capacidad de percibir a través de nuestros sentidos la belleza natural y artificial. De conmoverse en mayor o menor grado en función del entorno visual que observa, que además de ser un asunto individual y personal, está mediado por el contexto social en el que se desenvuelve el individuo. La cultura con la que estamos relacionados determina los valores criterios o estándares estéticos que caracterizan a la comunidad en la que vivimos.

El estudiante aprecia y valora los contextos de donde provienen; estos se desarrollan a partir del reconocimiento de contextos paisajísticos que se presentan en el territorio -área de estudio- relevando aquellos que tienen nombre propio.

La sensibilidad estética se aprecia en diferentes momentos del trabajo; cuando esquematizan ideas con originalidad, cuando formatean su lámina de trabajo con calidad compositiva, fuente: estilo, tamaño, color, etc.

\section{Formación ciudadana}

Según Tedesco (2017):

Las profundas transformaciones políticas, económicas y culturales que caracterizan a la sociedad actual han modificado radicalmente el contexto y los desafíos que enfrenta la formación ciudadana, al punto tal que se cuestiona incluso la idea misma de ciudadanía, que tiende a ser reemplazada por categorías tales como "cliente", "consumidor" o "usuario". Frente a estas opciones profundamente regresivas, se nos presenta el desafío de revitalizar la formación ciudadana orientada a la construcción de sociedades más justas, tarea más urgente que nunca cuando vemos cómo crecen las opciones racistas, xenófobas y fundamentalistas. (párr. 2)

La dimensión cognitiva de la nueva formación ciudadana no puede reducirse al conocimiento del funcionamiento de las instituciones democráticas (ellas mismas en 
procesos de profunda transformación) sino al conocimiento y la capacidad de analizar una enorme cantidad de informaciones y documentación. (párr.3)

El estudiante reconoce las posibilidades de participación como ciudadano en tanto se trata del territorio en sus diferentes ámbitos geográficos. Asimismo, es consciente que su participación con educación es un aporte a la democracia y a su permanencia.

Además, reconoce que las intervenciones en las que puede intervenir están provistas de métodos de análisis, evaluación y lineamientos para plantear propuestas de intervención.

\section{Comunicación}

Capacidad para comunicarse de manera efectiva a través del lenguaje oral y escrito, y del lenguaje técnico y computacional necesario para el ejercicio de la profesión.

Gómez Font (1999) dice:

$\mathrm{Si}$ algo caracteriza al lenguaje científico y al lenguaje técnico es su léxico. El léxico general, el propio de todos los hablantes, puede ser utilizado para transmitir mensajes a todos los que conocen una determinada lengua. Por el contrario, los textos especializados son los que contienen un vocabulario que sólo puede comprender un grupo muy reducido de hablantes, y todos los textos sobre ciencias o tecnología son así. Tratar de leer un texto especializado científico o técnico sin ser especialista en el campo correspondiente es casi lo mismo que tratar de leer un texto literario en una lengua que no se conoce.

En el lenguaje científico y técnico los vocablos especializados son absolutamente insustituibles y no pueden ser retirados del texto para colocar otros que actúen como sinónimos o casi sinónimos, pues estos no pueden existir. La parte esencial de lo que llamamos vocabulario especializado la constituye el léxico científico y técnico. La especialización no se produce por ningún otro mecanismo que pueda afectar al léxico sino por la eliminación de cualquier posibi- lidad significativa que no sea la deseada en la oportuna utilización del vocablo.

El lenguaje especializado exige un significante propio para cada significado. Un texto científico en el que cada noción especializada no tuviera una palabra (un significante) propia sería necesariamente un texto confuso. Sólo los especialistas pueden distinguir con precisión los términos propios de su ciencia, ya que frecuentemente estos tienen la forma de una palabra del léxico general, pero en el texto científico o técnico tienen un significado unívoco para su empleo especializado. Quien pretenda interpretar el sentido de las voces propias de un campo especializado, sin ser especialista, caerá en una confusión total, pues cometerá el error de tratar esos términos como si fueran palabras de la lengua general, y la realidad es que no tienen nada que ver con ellos.

El científico o el técnico escriben para pocas personas, es decir, únicamente para los que dominan la parcela de la ciencia de la que tratan sus escritos; incluso en las obras de divulgación científica el autor no puede prescindir de los términos propios del lenguaje científico-técnico. Actualmente la exposición científica para un público amplio se hace a base de la colaboración entre los científicos y los periodistas especializados de los grandes periódicos y las grandes agencias de información internacionales. Estos últimos, los periodistas, deben transformar el lenguaje científico en lenguaje periodístico, prestando atención a los niveles de los receptores de esos mensajes, que pueden ser científicos, personas cultivadas o público en general y que pueden variar según los países y las sociedades, precisando cada uno de ellos una determinada forma en la exposición, en los razonamientos y en el lenguaje.

La tarea del estudiante se centra en utilizar el vocabulario, términos y conceptos que corresponden a la temática que desarrolla y comprende que estos términos carecen de si- 
nónimos por ser términos propios del lenguaje científico-técnico.

El facilitador interviene constantemente para que el estudiante desarrolle un proceso mental que le permita establecer una asociación o vínculo para recordar los nuevos conceptos (nemotecnia).

El logro final consiste en que el estudiante comunique con fluidez el tema desarrollado con orden y utilizando los términos apropiados y precisos.

\section{Referencias}

Comisión de las Comunidades Europeas. (2005) "Propuesta de Recomendación del Parlamento Europeo y del Consejo" sobre las competencias clave para el aprendizaje permanente. Bruselas. Recuperado de http://www.europarl.europa. eu/meetdocs/2004_2009/documents / com/com_com\%282005\%290548_/com _ com\%282005\%290548_es.pdf

Gobierno Vasco (s.f.). Competencia para la autonomía e iniciativa personal. Recuperado de http://ediagnostikoak.net/edweb/cas/ materiales-informativos/ED_marko_teorikoak/ Autonomia_e_iniciativa_personal.pdf

Decreto Supremo N. ${ }^{\circ}$ 016-2015-MINEDU । Gobierno del Perú. Recuperado de https:// www.gob.pe/institucion/minedu/normaslegales/118310-016-2015-minedu

Delors, J. (1996). Informe a la UNESCO de la Comisión Internacional sobre la Educación para el siglo XXI, PRESIDIDA POR Jacques Delors. La Educación encierra un tesoro. Santillana, Ediciones UNESCO.

Ennis, R. H. (1985). A logical basis for measuring critical thinking skills, en Educational Leadership, $43(2)$, pp. 44-48.

Galdona, J. "La transversalidad en el Currículo". La formación de la estructura ética de la persona. Universidad Católica de Uruguay. Recuperado de https://www.oei.es/historico/valores2/ boletin 10a01.htm

Goffman. E. (1993). La presentación de la persona en la vida cotidiana. Buenos Aires, Argentina: Amorrortu.
Gómez Font, A. (1999). "Un nuevo lenguaje técnico" director del Departamento de Español Urgente de la Agencia EFE. En unidadenladiversidad.com. Resumen de una conferencia ofrecida durante el III Coloquio sobre lenguaje y comunicación celebrado en Caracas. Revista Latinoamericana de Comunicación. ISSN digital: 1390-924X ISSN impreso: 1390-1079.

Ley Universitaria-Minedu. Recuperado de www. minedu.gob.pe/reforma-universitaria/pdf/ley_ universitaria.pdf

Manas, R. (1972). Algunos correlatos perceptivos de la sensibilidad estética. Revista Latinoamericana de Psicología, 4(2), 221-226. Fundación Universitaria Konrad Lorenz. Bogotá, Colombia.

González Ramírez, N. y Mas, J. (2002) El nuevo concepto de cultura: La nueva visión del mundo desde la perspectiva del otro. Pensar Iberoamérica- Revista de Cultura. Venezuela.

Organización para la Cooperación y el Desarrollo Económico (2001), Agencia de los Estados Unidos para el Desarrollo Internacional (USAID). Resumen ejecutivo (p. 4). Recuperado de https:// www.oecd.org/mexico/Diagnostico-de-laOCDE-sobre-la-Estrategia-de-CompetenciasDestrezas-y-Habilidades-de-Mexico-ResumenEjecutivo.pdf

Pigozzi, M. (2004). The 10 Dimensions of Quality in Education. UNESCO-IBE Training Tools for Curriculum Development.

Tedesco, J. (2017). Reformular la formación ciudadana. Recuperado de https:// eldiariodelaeducacion.com/blog/2017/03/14/ reformular-la-formacion-ciudadana/

Tribunal Constitucional. STC 00017-2008-AI Resolución. Recuperado de: https://tc.gob.pe/ jurisprudencia/2013/00017-20 08-AI\%20 Resolucion.pdf 